This item was submitted to Loughborough's Research Repository by the author.

Items in Figshare are protected by copyright, with all rights reserved, unless otherwise indicated.

\title{
Focusing of ground vibrations generated by high-speed trains travelling at trans-Rayleigh speeds
}

PLEASE CITE THE PUBLISHED VERSION

http://dx.doi.org/10.1016/j.soildyn.2017.06.015

PUBLISHER

(C) Elsevier

VERSION

AM (Accepted Manuscript)

\section{PUBLISHER STATEMENT}

This work is made available according to the conditions of the Creative Commons Attribution-NonCommercialNoDerivatives 4.0 International (CC BY-NC-ND 4.0) licence. Full details of this licence are available at: https://creativecommons.org/licenses/by-nc-nd/4.0/

\section{LICENCE}

CC BY-NC-ND 4.0

\section{REPOSITORY RECORD}

Krylov, Victor V.. 2017. "Focusing of Ground Vibrations Generated by High-speed Trains Travelling at Transrayleigh Speeds". figshare. https://hdl.handle.net/2134/25786. 


\title{
Focusing of ground vibrations generated by high-
}

\section{speed trains travelling at trans-Rayleigh speeds}

\author{
Victor V. Krylov* \\ Department of Aeronautical and Automotive Engineering, \\ Loughborough University, \\ Loughborough, Leicestershire LE11 3TU, UK
}

\begin{abstract}
In the present paper, the effects of focusing of ground vibrations generated by high speed trains travelling at trans-Rayleigh speeds, i.e. under the condition of ground vibration boom, are considered theoretically. These effects are similar to the effects of focusing of sound waves radiated by supersonic aircraft. In particular, if a railway track has a bend, e.g. to provide the possibility of changing direction of train movement, the Rayleigh surface waves generated by high-speed trains under the condition of ground vibration boom may become focused. This results in concentration of their energy along a simple caustic line at one side of the track and in the corresponding increase in ground vibration amplitudes. The effect of focusing of Rayleigh waves may occur also if a train moves along a straight line with acceleration $a$ and its current speed $v(t)$ is higher than Rayleigh wave velocity in the ground. In the present paper, both the above-mentioned focusing mechanisms are investigated in detail using the Green's function formalism and the expressions for space-time distributions
\end{abstract}

\footnotetext{
* Corresponding author. E-mail address: V.V.Krylov@lboro.ac.uk
} 
of track-train-induced dynamic forces that take into account either track curvature or train acceleration. It is shown that in both these cases the effect of focusing can result in noticeable increase in generated ground vibrations. The obtained results are illustrated by numerical calculations.

Keywords: Ground vibrations; High-speed trains; Focusing.

\section{Introduction}

High-speed trains represent a convenient and environmentally friendly alternative to road and air transportation. The last two decades have been marked by rapid development of highspeed railway systems in many countries throughout the world. As many other means of transportation, high-speed trains are not free of environmental problems. In particular, ground vibrations generated by high-speed trains is one of the major environmental problems that must be mitigated to allow high-speed trains to be used in densely populated areas.

It is well known that, if train speeds increase, the intensity of railway-generated vibrations generally becomes larger. For modern high-speed trains the increase in generated ground vibrations is especially high when train speeds approach certain critical velocities of elastic waves propagating in a track-ground system, the most important of them being the velocity of Rayleigh surface wave in the supporting ground. As was theoretically predicted by the present author [1-4], if a train speed $v$ exceeds the Rayleigh wave velocity $c_{R}$ in supporting soil, a ground vibration boom occurs. This phenomenon is similar to a sonic boom for aircraft crossing the sound barrier, and it is associated with a very large increase in generated ground 
vibrations, as compared to the case of conventional trains. The existence of ground vibration boom has been later confirmed experimentally $[5,6]$, which implies that one can speak of 'supersonic' ('superseismic') or, more precisely, 'trans-Rayleigh' trains [7-9] in the same way as

people speak of supersonic aircraft. The increased attention to the problems of ground vibrations associated with high-speed trains is reflected in a growing number of theoretical and experimental investigations in this area (see, e.g. [10-18]).

The aim of the present paper is to investigate the effect of focusing of ground vibrations generated by high-speed trains that may take place under the condition of ground vibration boom, i.e. at trans-Rayleigh train speeds, even in a homogeneous ground. Some preliminary results on this topic have been reported earlier [19]. The two specific cases to be considered are focusing due to track curvature and focusing due to train acceleration, which are of the same physical nature as similar cases of focusing of a sonic boom from supersonic aircraft, sometimes called 'superbooms' [20-22]. It will be demonstrated that in both these cases the effect of focusing can result in noticeable increase in generated ground vibrations.

It should be noted that any properly designed high-speed line will be built to ensure that trans-Rayleigh conditions will never be reached at operational train speeds. Therefore, practical cases of the focusing discussed in this paper are highly unlikely to appear. In spite of this, it is essential that the physical phenomenon of focusing of railway-generated ground vibrations be properly described and fully understood.

\section{Outline of the theory of railway-generated ground vibrations}

The main mechanisms of railway-generated ground vibrations are the wheel-axle pressure onto the track, the effects of joints in unwelded rails, the dynamically induced forces of 
carriage- and wheel-axle vibrations excited due to unevenness of wheels and rails, as well as variations in sleeper-ground contact from sleeper to sleeper and lateral inhomogeneities in the ground. In this paper we consider only the first mentioned generation mechanism that is present even for ideally flat rails and wheels - namely, the quasi-static pressure of wheel axles onto the track, which is also responsible for railway-generated ground vibration boom. The quasi-static pressure generation mechanism results from load forces applied to the railway track from each wheel axle that cause downward deflections of the track. These deflections produce a wave-like motion along the track at speed of the train that results in a distribution of each axle load over all the rail sleepers involved in the track deflection distance. Each sleeper, in turn, acts as a vertical force applied to the ground during the time necessary for a deflection curve to pass through the sleeper. These vertical forces are considered as point forces applied to the ground's surface directly, so that the spatial distribution of the sleeper forces through the ballast is disregarded in the model. To determine the track deflection curve and thus the time dependence of the forces applied from each sleeper to the ground the system of track and ground is modelled as an Euler-Bernoulli beam resting on Winkler foundation.

According to the earlier developed general theory [1-4, 7-9], in order to calculate ground vibrations generated by a train due to the quasi-static pressure mechanism, one needs to take into account the superposition of waves generated by each elementary source of ground vibrations (sleeper) activated by wheel axles of all carriages, with the time and space differences between sources (sleepers) being taken into account. Using the Green's function of an elastic half space $G_{z z}(\rho, \omega)$, the frequency spectrum of the normal component of ground vibration velocity on the ground surface $v_{z}(x, y, \omega)$ can be written as

$$
v_{z}(x, y, \omega)=\int_{-\infty}^{\infty} \int_{-\infty}^{\infty} P\left(x^{\prime}, y^{\prime}, \omega\right) G_{z z}(\rho, \omega) d x^{\prime} d y^{\prime},
$$


where $P\left(x^{\prime}, y^{\prime}, \omega\right)$ is the Fourier spectrum of distributed dynamic forces acting from all sleepers to the ground, and $\rho$ is the distance from each sleeper to the point of observation characterised by the coordinates $x, y$. The expression for the Green's function in (1), in which we take into account only the contribution of generated Rayleigh waves, can be written at large distances in the form (see e.g. [9, 23, 24])

$$
G_{z z}(\rho, \omega)=D(\omega) \frac{1}{\sqrt{\rho}} \exp \left(i k_{R} \rho-\gamma k_{R} \rho\right)
$$

where

$$
D(\omega)=\frac{(-i \omega) q k_{R}{ }^{1 / 2} k_{t}{ }^{2}}{(2 \pi)^{1 / 2} \mu F^{\prime}\left(k_{R}\right)} \exp \left(-i \frac{3 \pi}{4}\right),
$$

and the factor $F^{\prime}\left(k_{R}\right)=\left.[d F(k) / d k]\right|_{k=k R}$ is a derivative of the so-called Rayleigh determinant

$$
F(k)=\left(2 k^{2}-k_{t}^{2}\right)^{2}-4 k^{2}\left(k^{2}-k_{t}^{2}\right)^{1 / 2}\left(k^{2}-k_{l}^{2}\right)^{1 / 2}
$$

taken at $k=k_{R}$. The structure of the function $P\left(x^{\prime}, y^{\prime}, \omega\right)$, i.e. the Fourier transform of the spatial distribution of time-dependent dynamic forces acting from sleepers to the ground, will be discussed below.

The notations in (1)-(4) are as follows: $\rho=\left[\left(x-x^{\prime}\right)^{2}+\left(y-y^{\prime}\right)^{2}\right]^{1 / 2}$ is the distance between the source (with current coordinates $x^{\prime}, y^{\prime}$ ) and the point of observation located on the surface (with the coordinates $x, y$ ), $\omega$ is a circular frequency, $k_{R}=\omega / c_{R}$ is the wavenumber 
of a Rayleigh surface wave, $c_{R}$ is the Rayleigh wave velocity, $k_{l}=\omega / c_{l}$ and $k_{t}=\omega / c_{t}$ are the wavenumbers of longitudinal and shear bulk elastic waves, where $c_{l}=\left[(\lambda+2 \mu) / \rho_{0}\right]^{1 / 2}$ and $c_{t}=\left(\mu \rho_{0}\right)^{1 / 2}$ are longitudinal and shear wave velocities, $\lambda$ and $\mu$ are the elastic Lame' constants, $\rho_{0}$ is mass density of the ground, $q=\left(k_{R}^{2}-k_{l}^{2}\right)^{1 / 2}$, and $\gamma=0.001-0.1$ is a nondimensional loss factor describing the attenuation of Rayleigh waves in soil.

As was mentioned above, function $P\left(x^{\prime}, y^{\prime}, \omega\right)$ describes the frequency spectrum of the spatial distribution of all load forces acting along the track. This spectrum can be found by taking a Fourier transform of the time and space dependent load forces $P\left(t, x^{\prime}, y^{\prime}\right)$ applied from the track to the ground. Note that the function $P\left(t, x^{\prime}, y^{\prime}\right)$ does not depend on possible layered structure of the ground and remains the same for both vertically homogeneous and inhomogeneous half spaces. In the model under consideration, all properties of track and train, which determine generation of ground vibrations, are described by the above mentioned function of load forces $P\left(t, x^{\prime}, y^{\prime}\right)$.

Being interested in fundamental features of the phenomenon of focusing of railwaygenerated Rayleigh waves, in this paper, for the sake of simplicity, we consider ground vibrations generated by a single axle load only. We recall that for a single axle load moving at speed $v$ along a straight track (located at $y=0$ ), the load function has the form [2, 9]:

$$
P\left(t, x^{\prime}, y^{\prime}=0\right)=\sum_{n=-\infty}^{\infty} P\left(t-x^{\prime} / v\right) \delta\left(x^{\prime}-n d\right) \delta\left(y^{\prime}\right)
$$

where $P\left(t-x^{\prime} / v\right)$ is the time-delayed dynamic force acting from a sleeper with a coordinate $x^{\prime}$ to the ground surface, and the delta-function $\delta\left(x^{\prime}-n d\right)$ takes the periodic distribution of sleepers into account, where $d$ is the periodic inter-sleeper distance. Using the expression for $P(t)$ (see $[4,8,9])$, taking the Fourier transform of (5) to calculate $P\left(x^{\prime}, y^{\prime}, \omega\right)$, and substituting 
the result into (1) using the expression for Green's function $G_{z z}(\rho, \omega)$ (see formulas (2)-(4)) results in the following expression for the vertical vibration velocity $v_{z}$ of Rayleigh waves generated on the ground surface $(z=0)$ at the point of observation with the coordinates $x, y$ by a single axle load moving along the straight track at speed $v$ :

$$
v_{z}(x, y, \omega)=P(\omega) D(\omega) \sum_{n=-\infty}^{\infty} \frac{1}{\sqrt{\rho_{n}}} \exp \left[i \frac{\omega}{v} n d+(i-\gamma) \frac{\omega}{c_{R}} \rho_{n}\right]
$$

Here $\rho_{n}=\left[(x-n d)^{2}+y^{2}\right]^{1 / 2}$ is the distance from the sleeper characterised by the number $n$ to the observation point. The function $P(\omega)$ in (6) has the following form (see $[4,8,9]$ for detail):

$$
P(\omega)=-\frac{12.8 \frac{T d}{v \pi^{2}}}{\frac{\omega^{4}}{\beta^{4} v^{4}}-4 \frac{\omega^{2}}{c_{\min }{ }^{2} \beta^{2}}-8 i \frac{\eta \omega}{c_{\min } \beta}+4},
$$

where $T$ is the axle load, $c_{\min }$ is the minimal phase velocity of track flexural waves propagating in a track/ground system (this velocity is related to $c_{R}$ via the parameters of Winkler foundation expressed in terms of the ground elastic parameters, and it is usually larger than $c_{R}$ by $\left.10-20 \%\right), \beta$ is the parameter dependent on the elastic properties of track and ground [8] and measured in $m^{-1}$, and $\eta$ is a non-dimensional track damping parameter. For relatively low train speeds, i.e., for $v<c_{R}$, the dynamic solution (7) for the force spectrum $P(\omega)$ goes over to the quasi-static one $[8,9]$. As train speeds increase and approach or exceed the minimal track wave velocity, the spectra $P(\omega)$ become broader and larger in amplitudes, and a second peak appears at higher frequencies. 
Note that the expression (7) has been obtained in the assumption that the ground supporting the track is considered as a linear elastic medium. Over the last two decades though, the increasing attention has been paid also to nonlinear behaviour of the supporting ground, especially in the case of trains travelling at or above track critical speeds [25-27].

For 'trans-Rayleigh trains', i.e. for trains travelling at speeds $v$ higher than Rayleigh wave velocity in the ground $c_{R}$, the analysis of the expression (6) shows that maximum radiation of ground vibrations takes place if the train speed $v$ is larger than Rayleigh wave velocity $c_{R}$ [1-4, 7-9]. Under this condition, a ground vibration boom takes place, i.e., ground vibrations are generated as quasi-plane Rayleigh surface waves symmetrically propagating at angles $\Theta=$ $\cos ^{-1}\left(c_{R} / v\right)$ in respect to the track, and with amplitudes much larger than in the case of conventional trains. This phenomenon is similar to the well-known phenomenon of sonic boom from supersonic aircraft.

Note that for trans-Rayleigh trains these symmetrically propagating Rayleigh surface waves are generated equally well on tracks with and without railway sleepers, whereas for conventional trains the presence of sleepers for the possibility of generation of Rayleigh waves is paramount. Without them no propagating waves are generated in the framework of the quasi-static pressure generation mechanism. This reflects the well-known fact that, if a point force of constant amplitude is moving at constant sub-Rayleigh speed along the ideally flat surface of an elastic half-space, there are no any generated elastic waves from such a force. Only quasi-static localised displacements are present that are moving along with the force. However, if the speed of the moving force exceeds the Rayleigh wave velocity, then Rayleigh waves are radiated. If the same force is moving along the railway track supported by discrete sleepers, Rayleigh waves are generated even at sub-Rayleigh load speeds (this is observed in practice via the presence of the so-called sleeper passage frequencies in the spectra of generated ground vibrations). For trans-Rayleigh trains, the amplitudes of generated 
Rayleigh waves grow substantially, and it does not matter in this case if the sleepers are present or not [2].

\section{Focusing due to track curvature}

If a track has a bend of radius $R$ to provide the possibility of changing direction of train movement, the wave fronts of Rayleigh ground waves generated under the condition of ground vibration boom may become concave at one side of the track, instead of being convex (under usual circumstances) or plane (under the condition of ground vibration boom). This may result in focusing of generated ground vibrations (Rayleigh surface waves) along a simple caustic line at one side of the track accompanied by the corresponding increase in their amplitudes. According to the geometrical acoustics approximation, this increase is up to infinity. However, because of the diffraction limit, the real increase for this type of caustic is much more modest and does not exceed 2-4 times.

It can be easily shown that if a train moves along a circular bend of radius $R$ at speed $v$ (with $v>c_{R}$ ) then the caustic line formed by rays of Rayleigh waves radiated at Mach angles $\Theta$ is a concentric circle of a smaller radius $r$ (see Fig. 1). Indeed, one can see from Fig. 1 that $r=R \sin \left(90^{\circ}-\theta\right)=R \cos \theta$. On the other hand, as it was mentioned in the previous section, under the condition of ground vibration boom $\cos \Theta=c_{R} / v$. Therefore, the radius $r$ of the concentric circle forming the caustic line is defined by the obvious simple expression:

$$
r=R \frac{C_{R}}{v}
$$


Note that, in the case of layered ground, generated Rayleigh waves become dispersive, i.e. $c_{R}$ $=c_{R}(\omega)$. Therefore, the position of the caustic in this case will depend on frequency, thus reducing the efficiency of focusing for Rayleigh waves described in a space-time domain.

The wave analysis of the above problem is rather straightforward and is based on the rewriting the expression for a distributed axle loads that takes into account the twodimensional geometry of a track with curvature. Namely, for a curved track, the expression (5) should be modified following the geometry of the curved track shown in Fig. 2.

As it follows from Fig. 2, $A B=C D=R \sin \varphi, \quad O C=R \cos \varphi, \quad$ and $D B=A C=O A-O C=R(1-\cos \varphi)$. Therefore, instead of the above-mentioned formula (5) for $P\left(t, x^{\prime}, y^{\prime}=0\right)$, the following expression for $P\left(t, x^{\prime}, y^{\prime}\right)$ should be used:

$$
P\left(t, x^{\prime}, y^{\prime}\right)=\sum_{n=-\infty}^{\infty} P\left(t-\frac{s^{\prime}}{v}\right) \delta\left(x^{\prime}-R \sin \frac{n d}{R}\right) \delta\left(y^{\prime}-R\left(1-\cos \frac{n d}{R}\right),\right.
$$

where the coordinate $s^{\prime}$ is measured along the curved track. Taking the Fourier transform of (9), substituting it into (1) and taking into account that for a sleeper with the number $n$ the angle $\varphi=\varphi_{m}=n d / R$, where $d$ is a sleeper periodicity, one can obtain the following formula for the spectral component of vertical vibration velocity $v_{z}(x, y, \omega)$ of Rayleigh waves generated on the ground surface at the point of observation with the coordinates $x, y$ by a single axle load moving along the curved track at speed $v$ :

$$
v_{Z}(x, y, \omega)=P(\omega) D(\omega) \sum_{n=-\infty}^{\infty} \frac{1}{\sqrt{\rho^{c} n}} \exp \left[i \frac{\omega}{v} n d+(i-\gamma) \frac{\omega}{c_{R}} \rho^{c}{ }_{n}\right] .
$$


Here the distance $\rho_{n}^{c}$ from the sleeper characterised by the number $n$ to the observation point is described by the formula:

$$
\rho^{c} n=\sqrt{\left[x-R \sin \left(\frac{n d}{R}\right)\right]^{2}+\left[y-R\left(1-\cos \left(\frac{n d}{R}\right)\right]^{2}\right.},
$$

and the other notations are the same as in the previous section.

The results of the calculations of the spatial distribution of ground vibration field $v_{z}(x, y, \omega)$ over the surface area of $75 \times 75 \mathrm{~m}$ (i.e. the wave snapshot shown in arbitrary units as a greyscale plot) generated at the frequency component $f=10 \mathrm{~Hz}$ by a single axle load travelling along the curved track with the radius of curvature $R=100 \mathrm{~m}$ are shown in Fig. 3 as a greyscale contour plot. The horizontal and vertical axes in Fig. 3 represent surface rectangular coordinates in normalised (non-dimensional) units changing from 0 to 1: $x_{n}=$ $x[\mathrm{~m}] / 75[\mathrm{~m}]$ and $y_{n}=y[\mathrm{~m}] / 75[\mathrm{~m}]$. The direction of travel is from left to right. The load speed is $v=50 \mathrm{~m} / \mathrm{s}$, and the velocity of Rayleigh wave in the ground was set as $c_{R}=45 \mathrm{~m} / \mathrm{s}$ (this corresponds to the value of Mach number $M=v / c_{R}=1.11$ ). Other relevant parameters were as follows: $d=0.7 \mathrm{~m}, \beta=1.28 \mathrm{~m}^{-1}$, which is typical for British railway tracks, and $\gamma=$ 0.001 .

It can be clearly seen from Fig. 3 that the wave fronts of generated Rayleigh waves become concave on the left-hand side of the track (in respect of the direction of travel), which results in focusing of Rayleigh waves along the caustic line accompanied by the increase in their amplitudes. Numerical values, that are not displayed here for clarity, show that the average increase in amplitudes due to focusing is about two times. Although this increase is 
rather small, one should keep in mind that it appears in addition to the already very large level of generated vibrations due to the ground vibration boom.

Figures 4 and 5 show the same results presented as a colour contour plot and as a greyscale surface plot respectively. Larger wave amplitudes in the area of focusing can be clearly seen in Fig. 4 from brighter red and blue areas corresponding to positive and negative vertical wave displacements. Figure 5, a three-dimensional surface plot, gives a direct representation of the wave amplitude distribution over the area by simply showing real vertical displacements. The locations of the curved track can be easily identified in Figs. 3-5. They are also shown by white dashed lines in Figs. 3 and 4.

Note that the radius of track curvature $R=100 \mathrm{~m}$, for which the results of Figs. 3-5 have been calculated, is rather small for real high-speed trains that require much larger radii of curvature to avoid derailment (e.g. for a train speed of $33 \mathrm{~m} / \mathrm{s}$ (or $120 \mathrm{~km} / \mathrm{h}$ ) the value of $R$ should be not less than $450 \mathrm{~m}$ ). However, using the unrealistically small value of $R=100 \mathrm{~m}$ for the example calculations presented in Figs. 3-5 helps to illustrate the phenomenon of focusing due to track curvature more clearly.

Figure 6 shows in greyscale the calculated spatial distribution of ground vibrations $v_{z}(x$, $y$, $\omega)$ over the surface area of $75 \times 75$ m generated by a single axle load travelling along the curved track with a more realistic larger radius of curvature $R=300 \mathrm{~m}$. All other parameters are the same as in Figs. 3-5. Like in Figs. 3-5, the location of the curved track can be easily identified in Fig. 6. One can also see from Fig. 6 that the focusing of generated Rayleigh waves, although less pronounced than in the case of $R=100 \mathrm{~m}$, is still clearly visible on the left from the curved track relative to the direction of travel. Amplification in the area of focusing is smaller in this case, about 1.3 times. 
Figure 7 presents a greyscale contour plot showing the calculated spatial distribution of ground vibrations $v_{z}(x, y, \omega)$ over the surface area of $75 \times 75 \mathrm{~m}$ generated by a single axle load travelling along the curved track with a very large radius of curvature $R=5000 \mathrm{~m}$. With such a large radius of curvature, the curved track is expected to behave as straight track for generation of ground vibrations within the surface area of 75 x 75 m near the track. All other parameters are the same as in the previous figures. One can see from Fig. 7 that, as expected, there is no focusing any more, and the spatial distributions of generated ground vibrations (Rayleigh waves) are perfectly symmetric in respect of the track, thus illustrating classical Mach angles formed by the flat wave fronts.

\section{Focusing due to train acceleration}

The effect of focusing of Rayleigh waves generated by high-speed trains may occur also in the case of a train accelerating along a straight line if its current speed, $v=v(t)=v_{0}+a t$, is higher than Rayleigh wave velocity in the ground $c_{R}$ (here $v_{0}$ is the initial train speed and $a$ is the acceleration). The geometrical acoustics consideration of this effect is illustrated in Fig. 8 for three different positions of a moving train characterised by the changed angles of radiation of Rayleigh wave rays - angles $\theta_{1}, \theta_{2}$ and $\theta_{3}$ respectively. One can see that the caustic line in this case is not confined to the area near the train path, but moves away from it as the train moves along the track and its speed increases.

To apply the wave approach to this problem, it is convenient to express the train current speed $v$ as a function of the distance $s$ measured along the track. It follows from the kinematics of motion of a particle with acceleration $a$ that 


$$
v=v_{0} \sqrt{1+\frac{2 a s}{v_{0}^{2}}}
$$

Expressing the distance as $s=n d$ and substituting the expression (12) for $v$ into (6) gives the following formula for ground vibration velocity generated by a single load moving with acceleration $a$ :

$$
v_{z}(x, y, \omega)=P(\omega) D(\omega) \sum_{n=-\infty}^{\infty} \frac{1}{\sqrt{\rho_{n}}} \exp \left[i \frac{\omega}{v_{0} \sqrt{1-2 a d n / v_{0}^{2}}} n d+(i-\gamma) \frac{\omega}{c_{R}} \rho_{n}\right] .
$$

The meaning of the other functions and parameters in the expression (13) remain the same as in the previous sections.

The results of calculations of the spatial distribution of ground vibrations over the area of $75 \times 75 \mathrm{~m}$ generated at the frequency component $f=10 \mathrm{~Hz}$ by a single axle load travelling along the straight track with a train acceleration $a=1 \mathrm{~m} / \mathrm{s}^{2}$ are shown in Fig. 9 in the form of a greyscale contour plot. The initial load speed was $v_{0}=50 \mathrm{~m} / \mathrm{s}$, and the velocity of Rayleigh wave in the ground was set as $c_{R}=45 \mathrm{~m} / \mathrm{s}$ (this corresponds to the initial value of Mach number $M_{0}=v_{0} / c_{R}=1.11$ ). Figure 10 presents the same results in the form of a colour contour plot.

It is seen from Figs. 9 and 10 that on both sides of the track the concave wave fronts of Rayleigh waves are formed symmetrically, and the focusing occurs, accompanied by some amplification of Rayleigh wave amplitudes in the focusing areas. Like in the case of curved tracks with the radius of curvature $R=100 \mathrm{~m}$, this amplification is rather moderate, about 
two times. Note that the focusing areas (caustics) are moving away from the track with the distance, in agreement with the geometrical acoustics consideration discussed above.

Figure 11 shows (in greyscale) the results of similar calculations of the spatial distribution of ground vibrations generated over the area of $75 \times 75 \mathrm{~m}$ by a single axle load travelling along the straight track with a smaller acceleration $a=0.255 \mathrm{~m} / \mathrm{s}^{2}$ (other parameters are the same as in Figs. 9 and 10). It can be seen from Fig. 11 that in this case of smaller acceleration the focusing is still present, but it becomes less pronounced, as expected. It is worth to remind the reader that focusing of railway-generated Rayleigh waves due to train acceleration affects both sides of a straight track, and it is symmetrical in respect of the track.

Note that the value of train acceleration $a=0.255 \mathrm{~m} / \mathrm{s}^{2}$ used in the above calculations is typical for French TGV trains, when they depart from railway terminals and move until they reach the maximum operational speeds. The same value of train acceleration is expected to be used for the proposed high-speed railway network HS2 in the UK. It should be noted that during braking the associated train deceleration may easily reach $0.5 \mathrm{~m} / \mathrm{s}^{2}$. However, as it follows from the above theory, focusing of train-induced ground vibrations can be caused only by train acceleration. Deceleration does not cause focusing because radiated wave fronts in this case are convex rather than concave.

Returning to acceleration, one should mention that, in order to reach the projected maximum operational speed (400 km/h for HS2), a train will need to pass a certain acceleration distance $s$. This distance can be easily calculated using Equation (12), in which one should put the initial velocity $v_{0}=0$, if a train departs from the railway terminal. Calculation for $a=0.255 \mathrm{~m} / \mathrm{s}^{2}$ gives the value of acceleration distance $s$ equal to $24.1 \mathrm{~km}$. This is a rather large distance that illustrates the fact that in densely populated countries, with relatively short distances between railway terminals, a significant proportion of the routes, sometimes 40-60\%, will be covered by trains travelling with acceleration and deceleration. 
This means that focusing of ground vibrations due to train acceleration can be a more frequent occasion than focusing due to track curvature.

\section{Conclusions}

It has been demonstrated in this paper that, for high-speed trains travelling along curved tracks at constant speeds that are larger than Rayleigh wave velocity in the ground (i.e. under the condition of ground vibration boom), the effect of track curvature may result in focusing of railway-generated ground vibrations (Rayleigh surface waves) at one side of the track and in the corresponding increase of their amplitudes.

The focusing effect for Rayleigh waves generated by high-speed trains may occur also in the case of a train accelerating along a straight line if its current speed is larger than Rayleigh wave velocity in the ground. In this case the focusing occurs symmetrically on both sides of the track.

It is unlikely that the described effects of focusing of railway-generated ground vibrations will make a detrimental impact on the environment. The reason for this is that railway operators try to avoid train operations at trans-Rayleigh speeds altogether. Nevertheless, it is important to understand that these effects should be taken into consideration, where appropriate, for more accurate predictions of ground vibration boom from high-speed trains. This applies mainly to 'sensitive' locations along the proposed high-speed railway routes characterised by low Rayleigh wave velocity in the supporting ground. Possible effects of focusing of generated ground vibrations, especially due to train acceleration, should be taken into account before considering suitable protection measures for these locations. 


\section{References}

[1] Krylov VV. On the theory of railway-induced ground vibrations. Journal de Physique IV 1994; 4 (C5): 769-772.

[2] Krylov VV. Generation of ground vibrations by superfast trains. Applied Acoustics 1995; 44: 149-164.

[3] Krylov VV. Vibrational impact of high-speed trains. I. Effect of track dynamics. Journal of the Acoustical Society of America 1996; 100(5): 3121-3134; Erratum, 1997; 101(6): 3810.

[4] Krylov VV. Effect of track properties on ground vibrations generated by high-speed trains. Acustica-acta acustica 1998; 84(1): 78-90.

[5] Madshus C, Kaynia AM. High speed railway lines on soft ground: dynamic behaviour at critical train speed. Proc. 6th International Workshop on Railway and Tracked Transit System Noise. Ile des Embiez, France 1998. p. 108-119.

[6] Adolfsson K, Andreasson B, Bengtsson P-E, Zackrisson P. High speed train X2000 on soft organic clay - measurements in Sweden. In: Barends FBJ et al., editors. Proceedings of the 12th European Conference on Soil Mechanics and Geotechnical Engineering. "Geotechnical Engineering for Transportation Infrastructure”. Amsterdam, The Netherlands, 7-10 June 1999. A.A. Balkema: Rotterdam: 1999. Vol. 3. p. $1713-1718$. 
[7] Krylov VV. Ground vibration boom from high-speed trains. Journal of Low Frequency Noise, Vibration and Active Control 1999: 18(4); 207-218.

[8] Krylov VV, Dawson AR, Heelis ME, Collop AC. Rail movement and ground waves caused by high-speed trains approaching track-soil critical velocities. Proceedings of the Institution of Mechanical Engineers, Part F: Journal of Rail and Rapid Transit 2000: 214; 107-116.

[9] Krylov VV. Generation of ground vibration boom by high-speed trains. In: Krylov VV, editor. Noise and vibration from high-speed trains. Thomas Telford Publishing: London: 2001. p. 251-283.

[10] Takemiya H. Ground vibrations alongside tracks induced by high-speed trains: prediction and mitigation. In: Krylov VV, editor. Noise and vibration from high-speed trains. Thomas Telford Publishing: London: 2001. p. 347-393.

[11] Degrande G, Schillemans L. Free field vibrations during the passage of a Thalys highspeed train at variable speed. Journal of Sound and Vibration 2001; 247(1): 131-144.

[12] Vostroukhov AV, Metrikine AV. Periodically supported beam on a visco-elastic layer as a model for dynamic analysis of a high-speed railway track. International Journal of Solids and Structures 2003; 40(21): 5723-5752.

[13] Takemiya H. Simulation of track-ground vibrations due to a high-speed train: the case of X2000 at Ledsgard. Journal of Sound and Vibration 2003; 261(3): 503-526.

[14] Sheng X, Jones CJC, Thompson DJ. A theoretical study on the influence of the track on train-induced ground vibration. Journal of Sound and Vibration 2004; 272: 909936. 
[15] Auersch L. The excitation of ground vibration by rail traffic: theory of vehicle-tracksoil interaction and measurements on high-speed lines. Journal of Sound and Vibration 2005; 284: 103-132.

[16] Galvin P, Dominguez J. High-speed train-induced ground motion and interaction with structures. Journal of Sound and Vibration 2007; 307: 755-777.

[17] Lombaert G, Degrande G. Ground-borne vibration due to static and dynamic axle loads of InterCity and high-speed trains. Journal of Sound and Vibration 2009; 319: 1036-1066.

[18] Xia H, Cao YM, De Roeck G. Theoretical modelling and characteristic analysis of moving-train induced ground vibrations. Journal of Sound and Vibration 2010; 329: 819-832.

[19] Krylov VV. Effects of the embankment topography and track curvature on ground vibration boom from high-speed trains. In: Grundmann H, Schueller GI, editors. "Structural Dynamics - EURODYN2002". Proceedings of the 4th International Conference on Structural Dynamics. Munich, Germany, 2-5 September 2002. A.A. Balkema Publishers: Lisse: 2002. Vol. 1, p. 473-478.

[20] Maglieri DJ, Plotkin KJ. Sonic boom. In: Aeroacoustics of flight vehicles: Theory and practice. Vol. 1: Noise sources. NASA: Langley Research Center: 1991. p. 519-561.

[21] Auger T, Coulouvrat F. Numerical simulation of sonic boom focusing. AIAA Journal 2002; 40(9): 1726-1734.

[22] Blumrich R, Coulouvrat F, Heimann D. Variability of focused sonic booms from accelerating supersonic aircraft in consideration of meteorological effects. Journal of the Acoustical Society of America 2005; 118(2): 696-706. 
[23] Graff KF. Wave motion in elastic solids. Clarendon Press: Oxford; 1975.

[24] Biryukov SV, Gulyaev YV, Krylov VV, Plessky VP. Surface acoustic waves in inhomogeneous media. Springer-Verlag: Berlin; 1995.

[25] Madshus C, Kaynia AM. High-speed railway lines on soft ground: dynamic behaviour at critical train speed. Journal of Sound and Vibration 2000; 231(3): 689-701.

[26] Alves Costa P, Calçada R, Silva Cardoso A. Bodare A. Influence of soil non-linearity on the dynamic response of high-speed railway tracks. Soil Dynamics and Earthquake Engineering 2010; 30(4): 221-235.

[27] Banimahd M, Woodward P, Kennedy J, Medero G. Three-dimensional modelling of high speed ballasted railway tracks. Proceedings of the Institution of Civil Engineers: Transport 2013; 166(TR2): 113-123. 


\section{Figure captions}

Fig. 1. Geometrical acoustics explanation of the focusing of Rayleigh waves radiated by a train (a single axle load in this example) travelling at speed $v$ along a curved track of radius $R$ under the condition of ground vibration boom $\left(v>c_{R}\right)$. The focusing occurs along the caustic line formed by a concentric circle of smaller radius $r$.

Fig. 2. Geometry of the problem of the curved track.

Fig. 3. Spatial distribution of ground vibration field (in arbitrary units, greyscale contour plot) generated over the area of $75 \times 75 \mathrm{~m}$ by a single axle load travelling at speed $v$ $=50 \mathrm{~m} / \mathrm{s}$ on a curved track (shown by a white dashed line) with the radius of curvature $R=100 \mathrm{~m}$.

Fig. 4. Spatial distribution of ground vibration field (the same as in Fig. 3, but presented as a colour contour plot) generated by a single axle load travelling at speed $v=50 \mathrm{~m} / \mathrm{s}$ on a curved track (shown by a white dashed line) with the radius of curvature $R=$ $100 \mathrm{~m}$.

Fig. 5. Spatial distribution of ground vibration field (the same as in Fig.3, but presented as a greyscale surface plot) generated by a single axle load travelling at speed $v=50$ $\mathrm{m} / \mathrm{s}$ on a curved track with the radius of curvature $R=100 \mathrm{~m}$. 
Fig. 6. Spatial distribution of ground vibration field (in arbitrary units, greyscale contour plot) generated over the area of $75 \times 75 \mathrm{~m}$ by a single axle load travelling at speed $v$ $=50 \mathrm{~m} / \mathrm{s}$ on a curved track with the radius of curvature $R=300 \mathrm{~m}$.

Fig. 7. Spatial distribution of ground vibration field (in arbitrary units, greyscale contour plot) generated over the area of $75 \times 75 \mathrm{~m}$ by a single axle load travelling at speed $v$ $=50 \mathrm{~m} / \mathrm{s}$ on a curved track with the radius of curvature $R=5000 \mathrm{~m}$.

Fig. 8. Geometrical acoustics explanation of the focusing of Rayleigh waves radiated by a train (a single axle load in this example) travelling with constant acceleration $a$ along a straight track under the condition of ground vibration boom $\left(v>c_{R}\right)$. The focusing occurs along the caustic line that is moving away from the track as the train passes by with acceleration.

Fig. 9. Spatial distribution of ground vibrations (in arbitrary units, greyscale contour plot) generated over the area of $75 \times 75 \mathrm{~m}$ by a single load travelling on a straight track (shown by a white dashed line) with acceleration $a=1 \mathrm{~m} / \mathrm{s}^{2}$ and initial train speed $v_{0}=50 \mathrm{~m} / \mathrm{s}$.

Fig. 10. Spatial distribution of ground vibrations (the same as in Fig. 9, but presented as a colour contour plot) generated by a single load travelling on a straight track (shown by a white dashed line) with acceleration $a=1 \mathrm{~m} / \mathrm{s}^{2}$ and initial train speed $v_{0}=50 \mathrm{~m} / \mathrm{s}$. 
Fig. 11. Spatial distribution of ground vibrations (in arbitrary units, greyscale contour plot) generated over the area of $75 \times 75 \mathrm{~m}$ by a single load travelling on a straight track with acceleration $a=0.255 \mathrm{~m} / \mathrm{s}^{2}$ and initial train speed $v_{0}=50 \mathrm{~m} / \mathrm{s}$. 


\section{Figures}

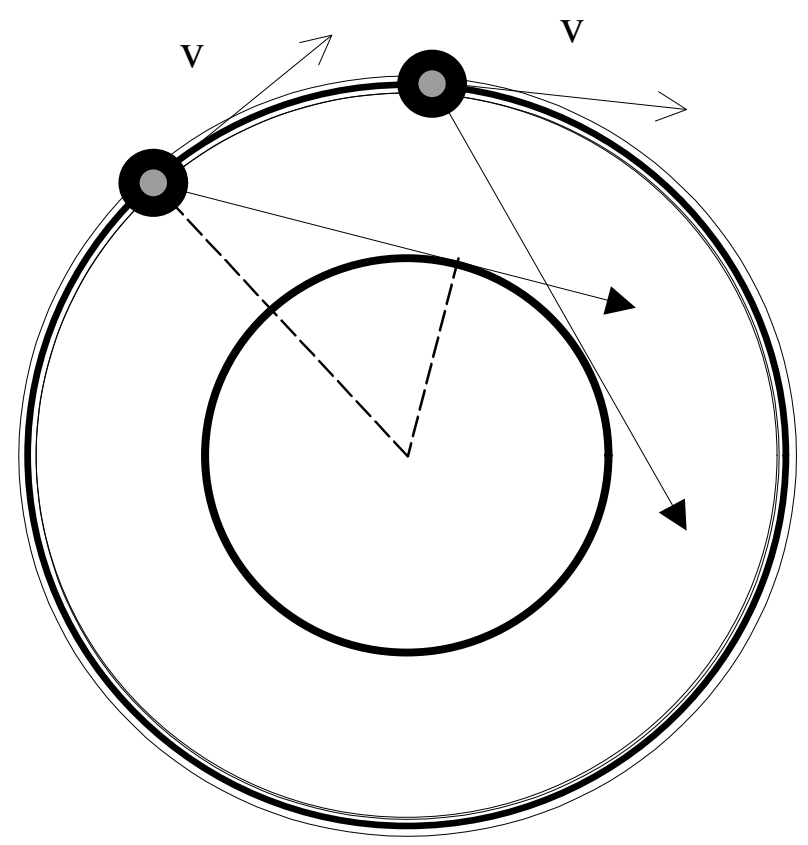

Fig. 1. Geometrical acoustics explanation of the focusing of Rayleigh waves radiated by a train (a single axle load in this example) travelling at speed $v$ along a curved track of radius $R$ under the condition of ground vibration boom ( $v>$ $\left.c_{R}\right)$. The focusing occurs along the caustic line formed by a concentric circle of smaller radius $r$. 


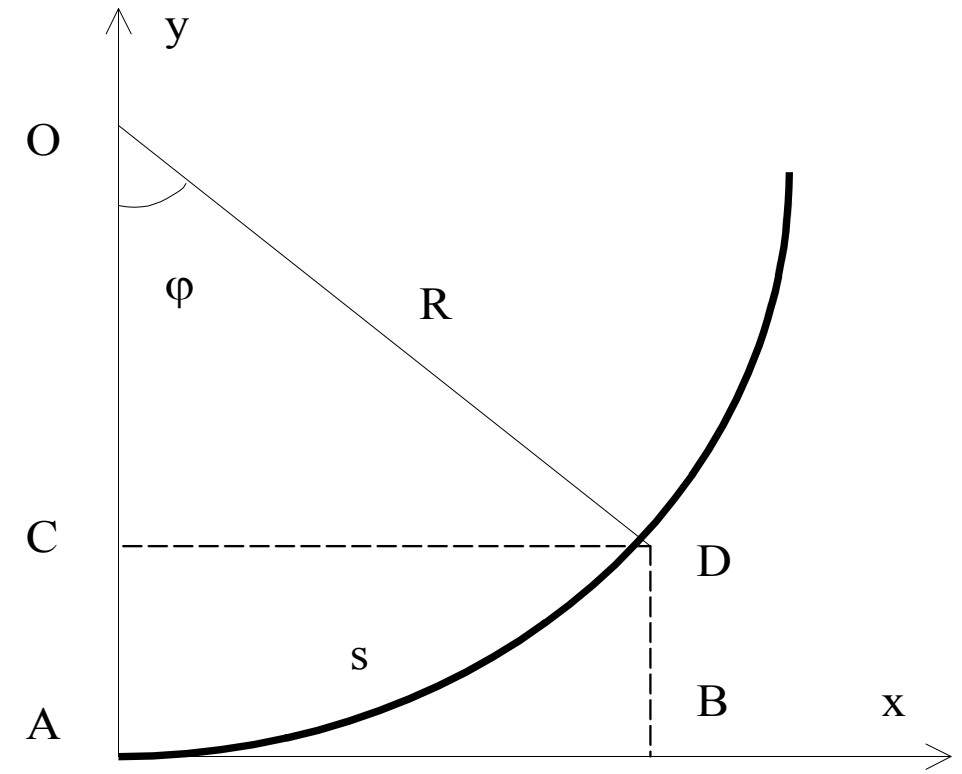

Fig. 2. Geometry of the problem of the curved track. 


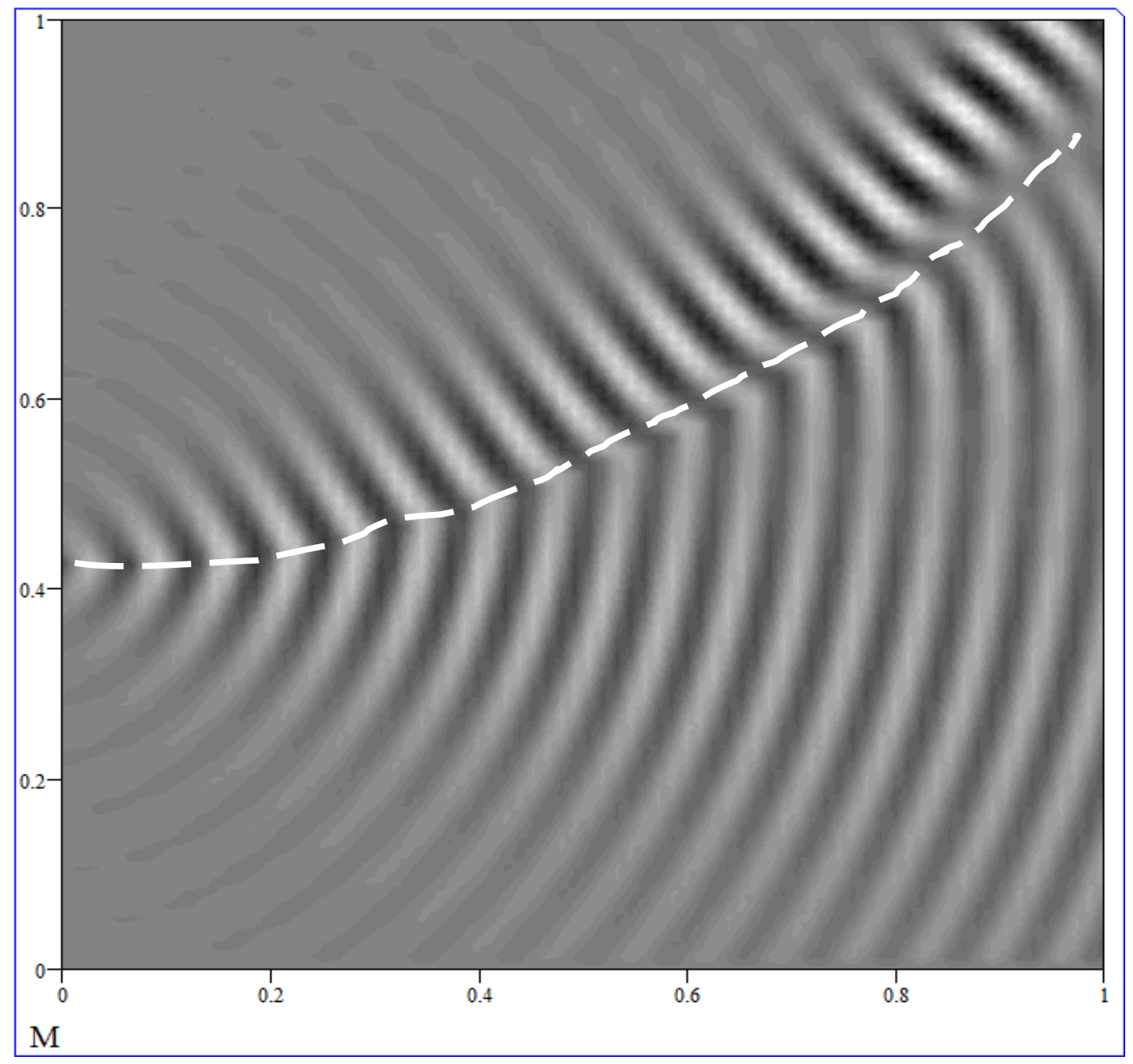

Fig. 3. Spatial distribution of ground vibration field (in arbitrary units, greyscale contour plot) generated over the area of $75 \times 75 \mathrm{~m}$ by a single axle load travelling at speed $v=50 \mathrm{~m} / \mathrm{s}$ on a curved track (shown by a white dashed line) with the radius of curvature $R=100 \mathrm{~m}$. 


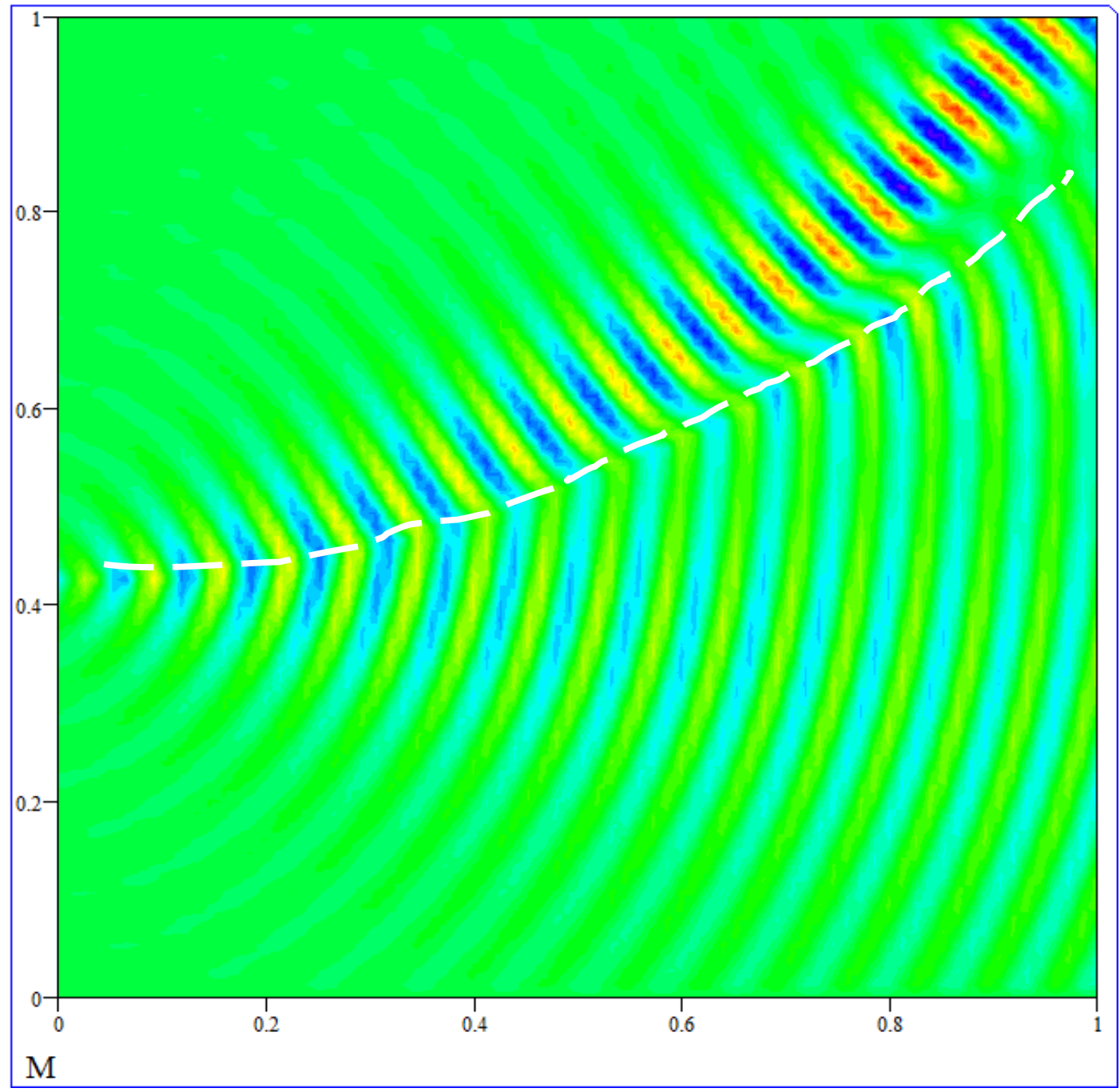

Fig. 4. Spatial distribution of ground vibration field (the same as in Fig. 3, but presented as a colour contour plot) generated by a single axle load travelling at speed $v=50 \mathrm{~m} / \mathrm{s}$ on a curved track (shown by a white dashed line) with the radius of curvature $R=100 \mathrm{~m}$. 


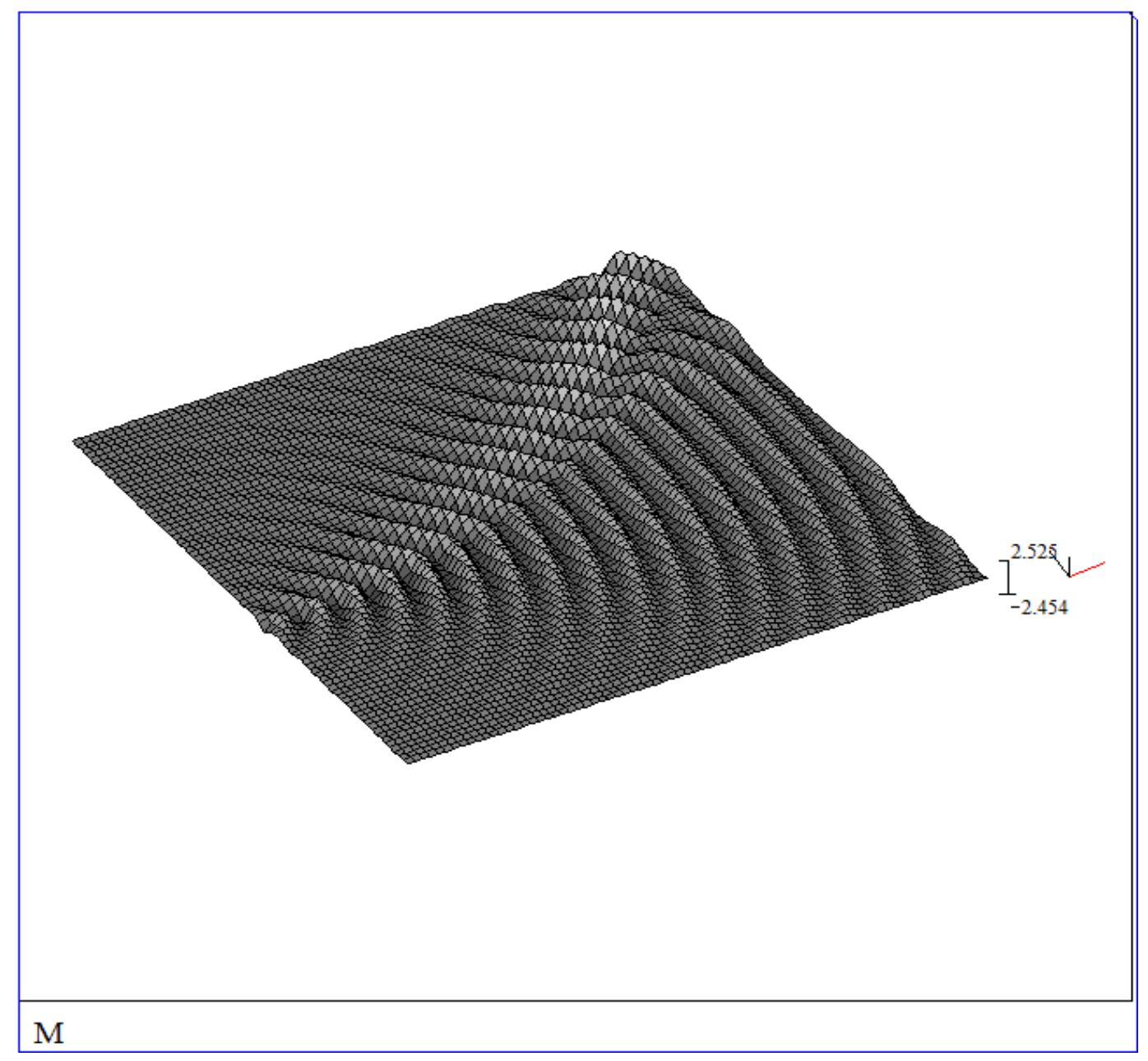

Fig. 5. Spatial distribution of ground vibration field (the same as in Fig.3, but presented as a greyscale surface plot) generated by a single axle load travelling at speed $v=50 \mathrm{~m} / \mathrm{s}$ on a curved track with the radius of curvature $R=100 \mathrm{~m}$. 


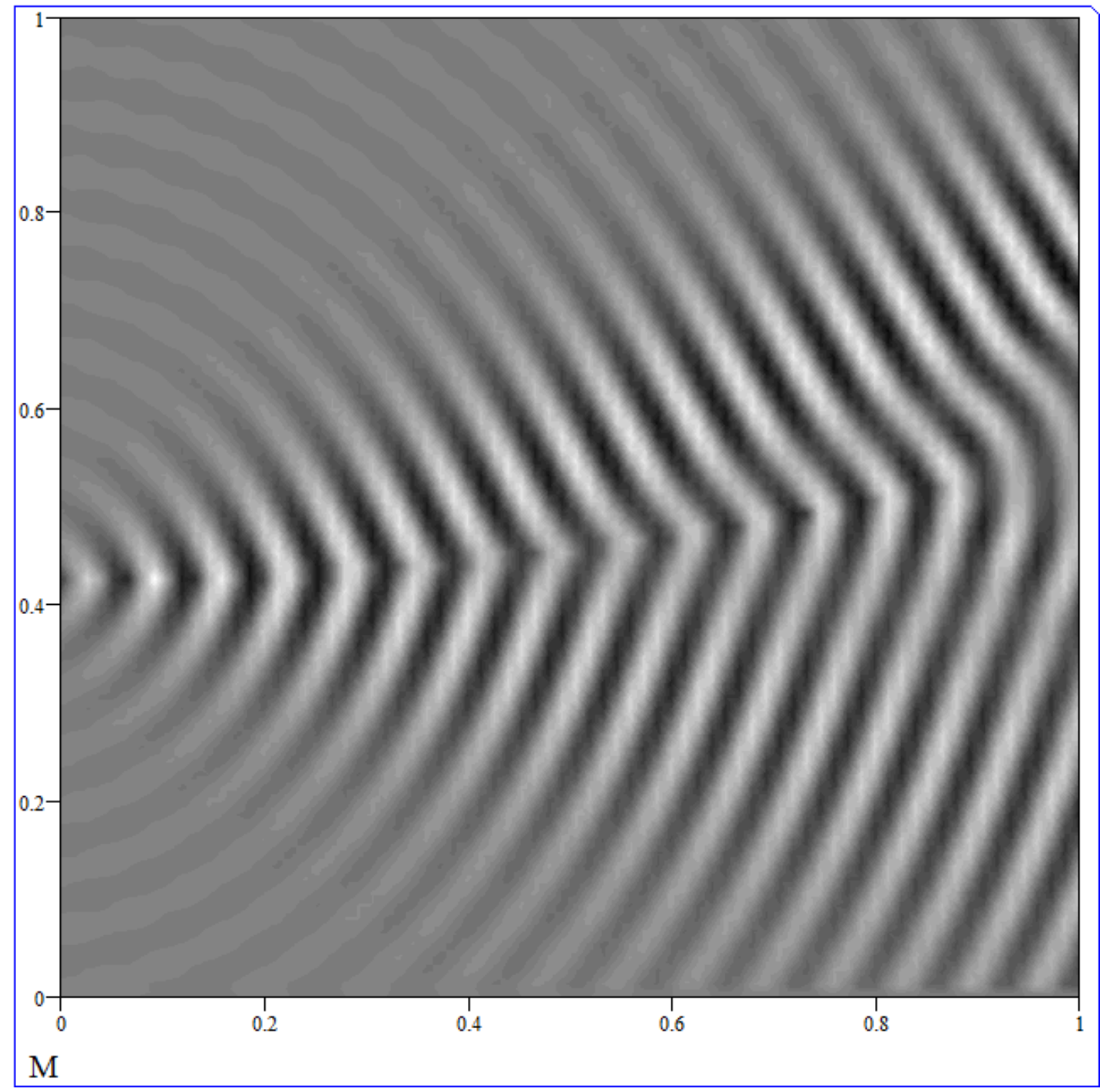

Fig. 6. Spatial distribution of ground vibration field (in arbitrary units, greyscale contour plot) generated over the area of $75 \times 75 \mathrm{~m}$ by a single axle load travelling at speed $v=50 \mathrm{~m} / \mathrm{s}$ on a curved track with the radius of curvature $R=300 \mathrm{~m}$. 




Fig. 7. Spatial distribution of ground vibration field (in arbitrary units, greyscale contour plot) generated over the area of $75 \times 75 \mathrm{~m}$ by a single axle load travelling at speed $v=50 \mathrm{~m} / \mathrm{s}$ on a curved track with the radius of curvature $R=5000 \mathrm{~m}$. 


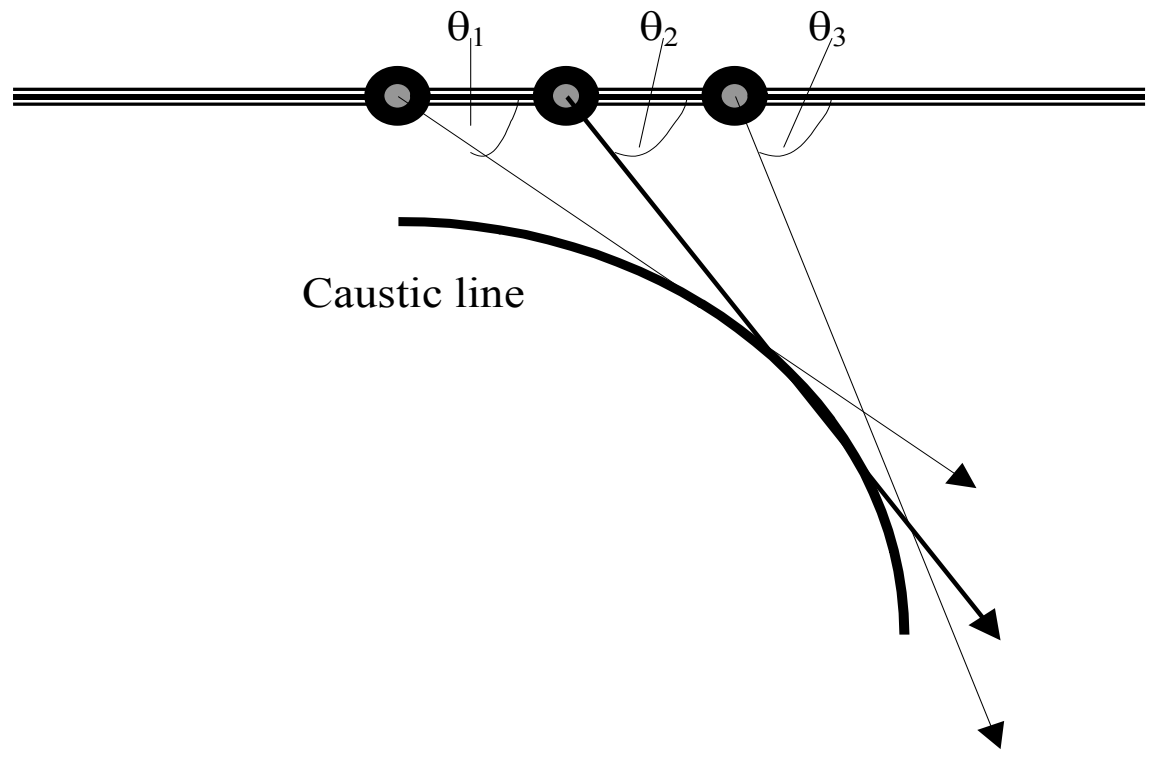

Fig. 8. Geometrical acoustics explanation of the focusing of Rayleigh waves radiated by a train (a single axle load in this example) travelling with constant acceleration $a$ along a straight track under the condition of ground vibration boom $\left(v>c_{R}\right)$. The focusing occurs along the caustic line that is moving away from the track as the train passes by with acceleration. 




Fig. 9. Spatial distribution of ground vibrations (in arbitrary units, greyscale contour plot) generated over the area of $75 \times 75 \mathrm{~m}$ by a single load travelling on a straight track (shown by a white dashed line) with acceleration $a=1 \mathrm{~m} / \mathrm{s}^{2}$ and initial train speed $v_{0}=50 \mathrm{~m} / \mathrm{s}$. 


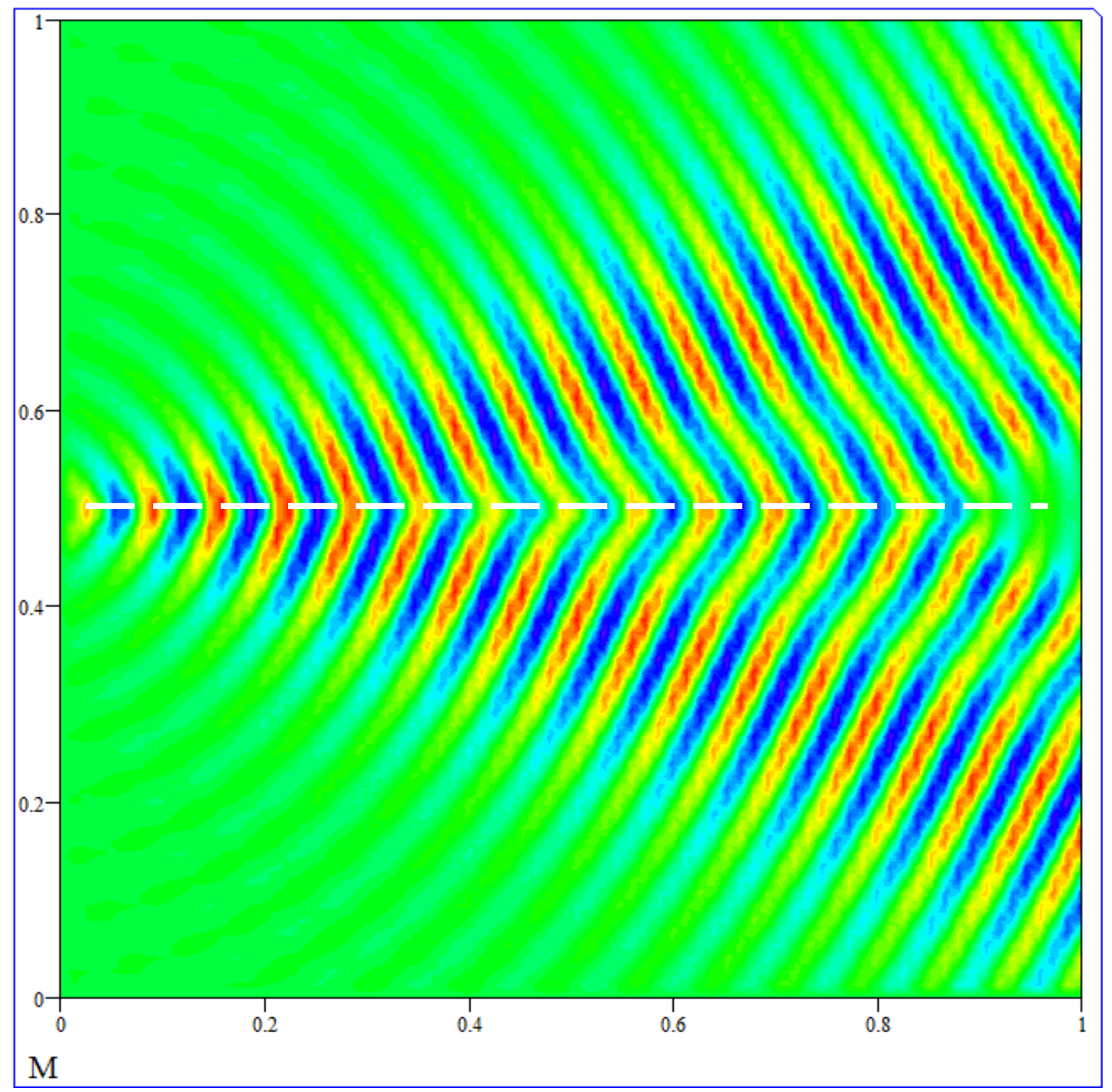

Fig. 10. Spatial distribution of ground vibrations (the same as in Fig. 9, but presented as a colour contour plot) generated by a single load travelling on a straight track (shown by a white dashed line) with acceleration $a=1 \mathrm{~m} / \mathrm{s}^{2}$ and initial train speed $v_{0}=50 \mathrm{~m} / \mathrm{s}$. 


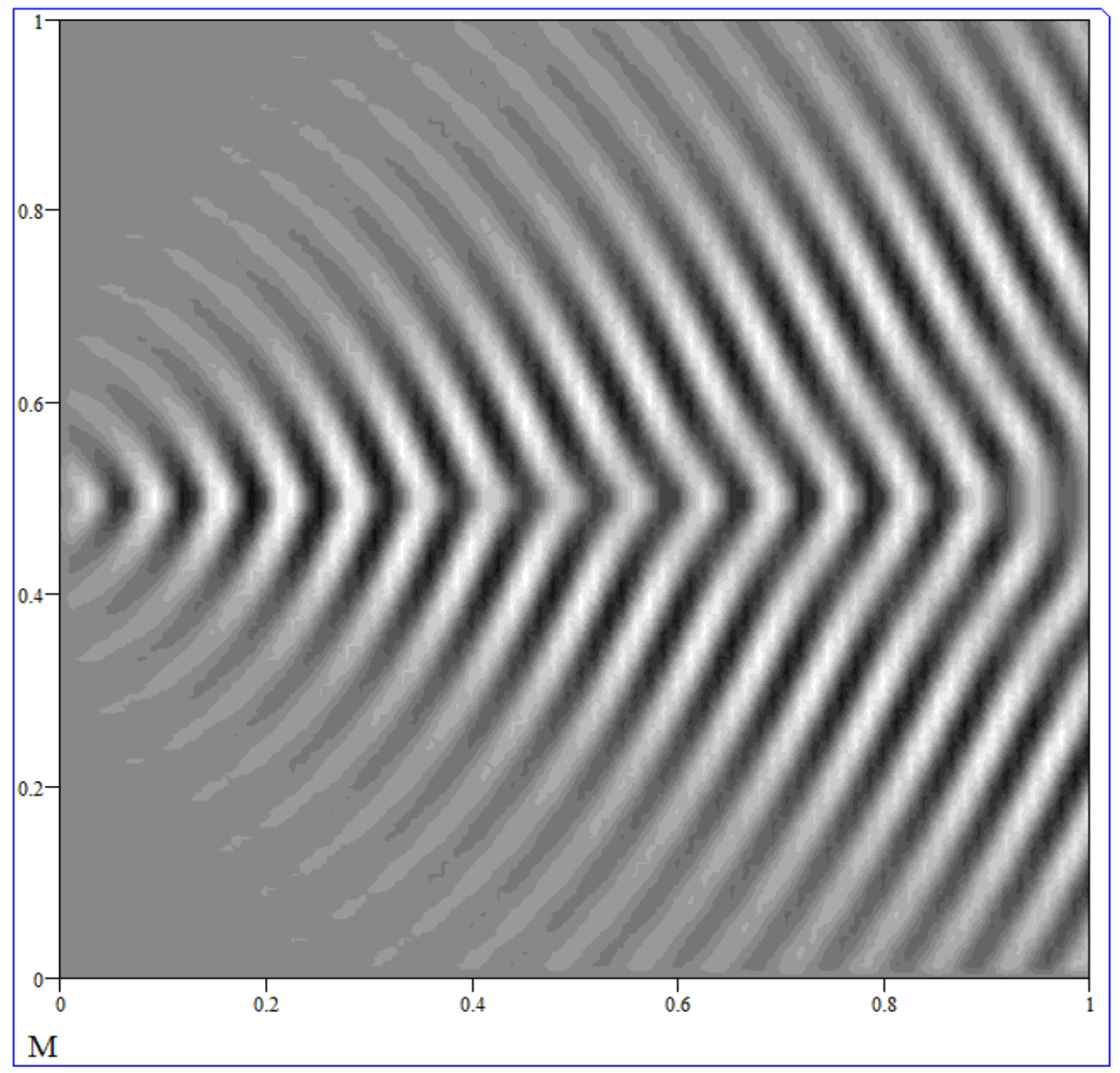

Fig. 11. Spatial distribution of ground vibrations (in arbitrary units, greyscale contour plot) generated over the area of $75 \times 75 \mathrm{~m}$ by a single load travelling on a straight track with acceleration $a=0.255 \mathrm{~m} / \mathrm{s}^{2}$ and initial train speed $v_{0}=50 \mathrm{~m} / \mathrm{s}$. 